\title{
Performance and Perspectives of Hadron Storage Rings
}

\author{
Andreas Lehrach ${ }^{* \dagger}$ \\ Institut für Kernphysik, Forschungszentrum Jülich, Germany \\ E-mail: a.lehrachefz-juelich.de
}

\begin{abstract}
Next generation hadron storage rings are seeking for highest intensity/luminosity, degree of polarization and precision. Advanced beam- and spin dynamics simulations are key tools to reach the required performance. In this paper the performance, upgrade plans and future perspectives of several medium-energy storage ring projects are discussed.
\end{abstract}

8th International Conference on Nuclear Physics at Storage Rings-Stori11, October 9-14, 2011

Laboratori Nazionali di Frascati, Italy

\footnotetext{
* Speaker.

${ }^{\dagger}$ The author is grateful to all members of the FAIR project team, HESR consortium, PAX collaboration, ENC study group, and the COSY machine group for their substantial contributions to this paper.
} 


\section{Introduction}

Hadron storage rings are used world-wide for various research topics and scientific applications (e.g., exploration of QCD physics in a wide energy range). Several medium-energy storage rings are either proposed, shortly before start of construction or successfully operated. The project status of the Facility for Antiproton and Ion Research (FAIR) and the High-Energy Storage Ring (HESR) is discussed, together with future Collider upgrade options of the HESR. Furthermore, the upgrade program for the Cooler Synchrotron COSY and perspectives for high-precision measurements of charged particle EDMs (electric dipole moment) in storage rings at Jülich are presented.

\section{Facility for Antiproton and Ion Research}

The FAIR Facility will provide antiproton and ion beams with unprecedented intensity and quality. In the final construction FAIR consists of up to eight accelerator rings, two linear accelerators and various beam transport lines [1]. The planned proton-linac together with the existing GSI accelerators serve as injector for this new facility. Utilizing the new synchrotron ring SIS100 intense beams of secondary beams - unstable nuclei or antiprotons - can be produced. Several cooler storage rings substantially increase the quality of these secondary beams in terms of energy spread and emittance. The Modularized Start Version is a stepwise approach to the realization of FAIR (see Fig. 1) [2] .

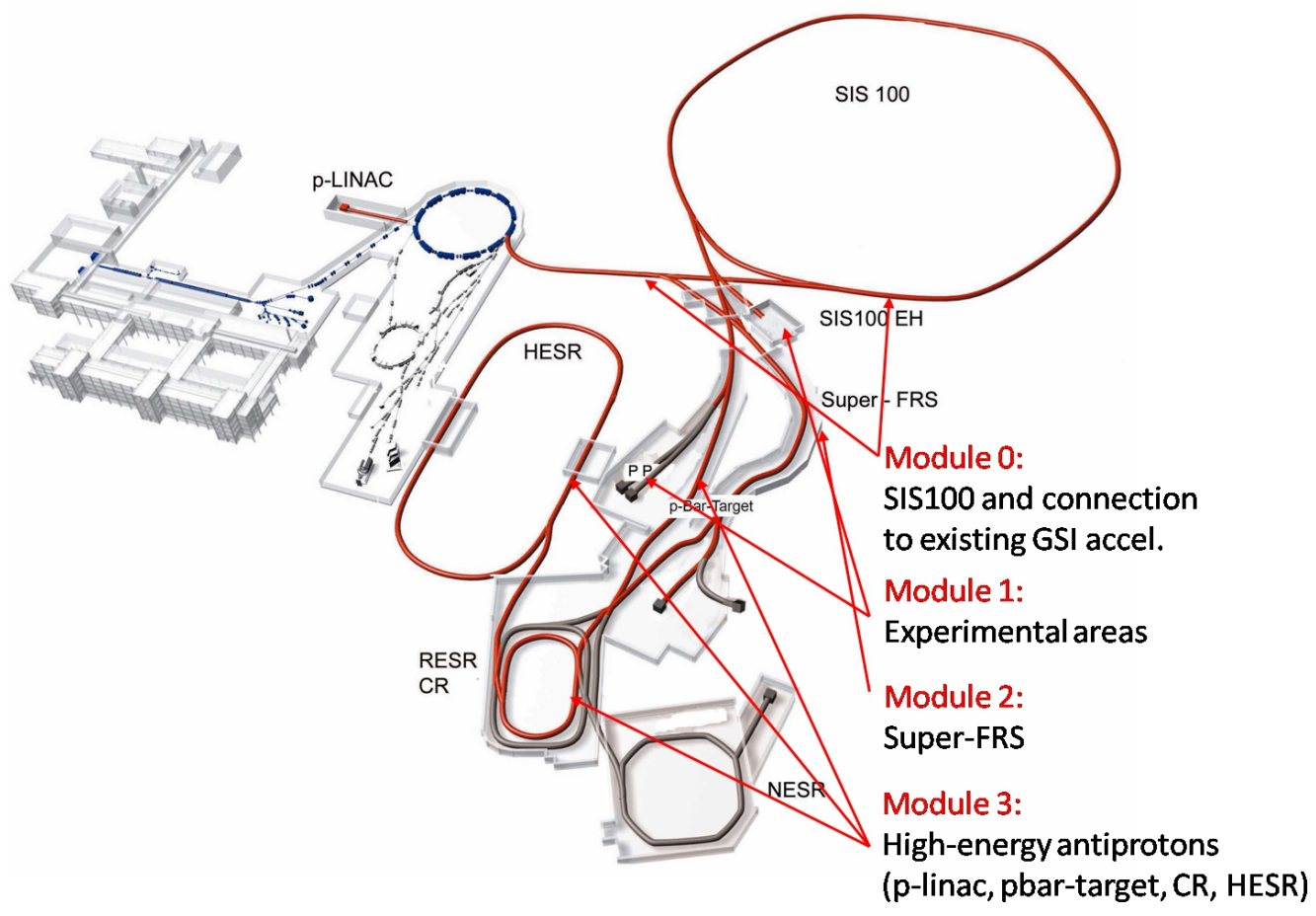

Figure 1: FAIR Modularized Start Version. Modules 0 to 3 are marked in red, modules 4 and 5 are not marked in color. On the left hand side of the figure the existing GSI facility is shown. 
It is structured in six modules:

- Module 0: Heavy-Ion Synchrotron SIS100, required for all particle beam species.

- Module 1: Experimental areas: CBM/HADES cave, experimental hall for APPA and detector calibrations.

- Module 2: Super-FRS for NuSTAR.

- Module 3: Antiproton facility for PANDA, providing further options also for NuSTAR ring physics.

Modules 4 and 5 are obvious upgrades of the Modularized Start Version. They will further strengthening the long-term scientific program of FAIR.

- Module 4: Second cave for NuSTAR, NESR storage ring for NuSTAR and APPA, building for antimatter program FLAIR.

- Module 5: RESR storage ring for higher beam intensity for PANDA and parallel operation with NuSTAR.

Presently civil construction for FAIR enters into the realization phase.

\section{High-Energy Storage Ring}

The HESR (see Fig. 2) is an essential part of the antiproton physics program at FAIR. It will provide antiprotons in the momentum range from 1.5 to $15 \mathrm{GeV} / \mathrm{c}$ for the internal target experiment PANDA [3]. A consortium consisting of FZ Jülich (as leading institution), GSI Darmstadt, Universität Mainz and ICPE-CA Bucharest is in charge of HESR design and construction. An important feature of this new facility is the combination of phase space cooled beams and thick internal targets (e.g., pellet targets) which results in demanding beam parameter requirements for two operation modes: high luminosity mode with peak luminosities of up to $2 \cdot 10^{32} \mathrm{~cm}^{-2} \mathrm{~s}^{-1}$ and high resolution mode with a beam momentum spread down to $10^{-5}$.

\subsection{Project Status}

Various beam dynamics studies have been performed to guarantee the required equilibrium beam parameters, beam lifetime and beam stability [4]. Powerful beam cooling is needed to reach demanding experimental requirements in terms of luminosity and beam quality. The design work of the HESR is in the final stage and the construction phase can start together with FAIR construction.

Beam dynamics simulations:

A chromaticity correction scheme has been developed for the HESR and optimized through dynamic aperture calculations. The estimated field errors of the HESR dipole and quadrupole magnets have been included in nonlinear beam dynamics studies. The ion optical settings of the HESR 


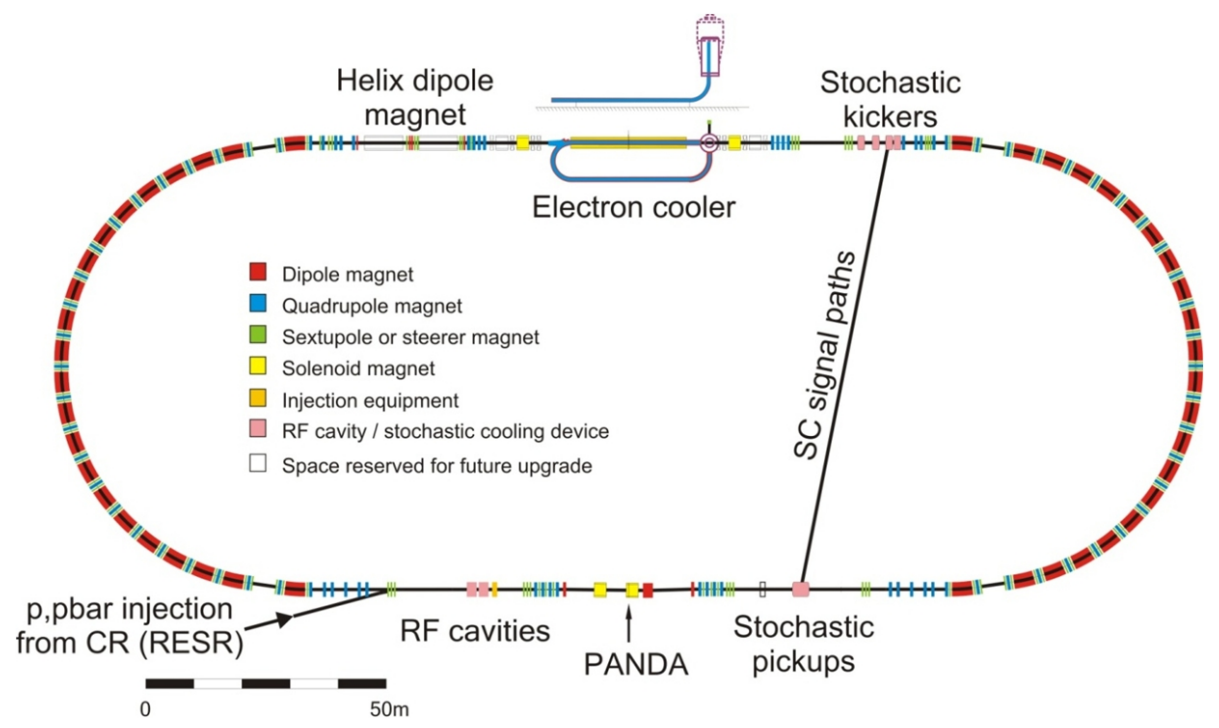

Figure 2: Schematic view of the HESR. Positions for injection, cooling devices and experimental installations are indicated. The upper straight is housing electron cooler, stochastic kickers, and space for a future upgrade. The lower straight contains injection, RF cavities, PANDA with target, and stochastic pickups.

have been improved using dynamic aperture calculations and the technique of frequency map analysis [5]. The accumulation and clearing of ion trapping in HESR has also been studied in detail [6]. Positively charged ions are produced by the interaction of the antiproton beam with residual gas and are continuously trapped in the negative potential well of the circulating antiproton beam. The removal of ions can be achieved using clearing electrodes and resonant beam shaking together with a broadband barrier bucket cavity. Comprehensive beam dynamic experiments have been carried out to test the developed momentum cooling models [7]. The interaction of the antiproton beam with an internal target and the fields of a barrier bucket cavity are included. The accumulator ring RESR is part of an upgrade program and only the collector ring CR is going to be available for antiproton collection and beam cooling from the beginning. Therefore a new injection and accumulation scheme for HESR has been developed utilizing a barrier bucket cavity and stochastic cooling [8].

Hardware development:

Magnet design of dipole, quadrupole, sextupole and correction dipole magnets has been finalized. Three-dimensional field calculations have been performed to minimize the multipole components of the various magnet types [9]. A detailed concept for the vacuum system of the HESR has been worked out [10].

Accelerator component tests and beam dynamics studies at COSY:

Studies of beam behavior with pellet target, barrier bucket and stochastic cooling have been performed at COSY [7]. Strong mean energy loss induced by the interaction of the beam with an internal pellet target cannot be compensated by beam cooling alone. To compensate the mean en- 
ergy loss and thus to provide an antiproton beam with a significantly reduced momentum spread, a broadband barrier bucket cavity will be used in the HESR [11]. Design, production and assembly of a barrier bucket cavity have been finalized. Meanwhile the barrier bucket cavity is routinely operated in COSY. New high-sensitivity pickups for stochastic beam cooling have been designed and built for the HESR. They have been successfully tested with COSY beam and have been proven their predicted performance.

\subsection{HESR Upgrade Options}

\subsubsection{Proton-Antiproton Collider}

Two experimental groups (ASSIA and PAX) expressed interest in proton-antiproton spin physics experiments utilizing the HESR. This requires production of polarized protons in a state-of-the-art polarized ion-source and advanced techniques to produced polarized antiprotons. For polarized antiprotons a dedicated Antiproton-Polarizer Ring (AP) with a large acceptance angle is needed in order to polarize antiprotons to a high polarization degree via the spin-filter method [12]. To preserve polarization during acceleration additional devices like fast tune-jump quadrupoles and Siberian snakes have to be implemented into several machines of the acceleration chain. Two different concepts have been worked out to finally collide this two polarized beams, a symmetric and an asymmetric collider (asymmetric version see Fig. 3) [13, 14]. The expected luminosities for the proposed collider configurations are ranging from 1 to $6 \cdot 10^{31} \mathrm{~cm}^{-2} \mathrm{~s}^{-1}$.

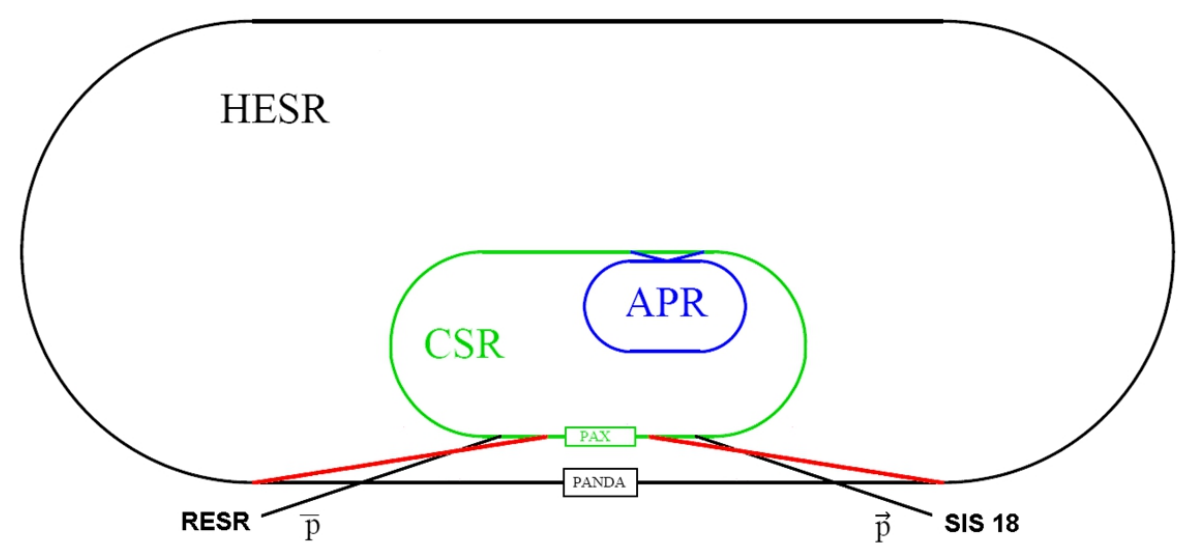

Figure 3: The proposed accelerator setup at the HESR (black), with the equipment used by the PAX collaboration: CSR (green), APR, beam transfer lines and polarized proton injector (all blue), two transfer lines (red), an asymmetric collider is setup (not to scale).

\subsubsection{Electron-Nucleon Collider}

The feasibility of a polarized Electron-Nucleon Collider (ENC) (see Fig. 4) with a centerof-mass energy up to $13.5 \mathrm{GeV}$ for luminosities up to $6 \cdot 10^{32} \mathrm{~cm}^{-2} \mathrm{~s}^{-1}$ is presently being studied [15]. The proposed concept integrates the planned $15 \mathrm{GeV} / \mathrm{c}$ HESR of the FAIR project for protons/deuterons and an additional $3 \mathrm{GeV} / \mathrm{c}$ electron ring [16]. Beam simulations of cooled beam equilibria concerning intra-beam scattering and beam-beam interaction have been performed for 
protons and deuterons [17]. For polarized proton/deuteron beams, additional equipment has to be installed in the HESR and several pre-accelerators of its injection chain to preserve the beam's polarization [18]. The concept for a polarized electron booster and storage ring is still under investigation.

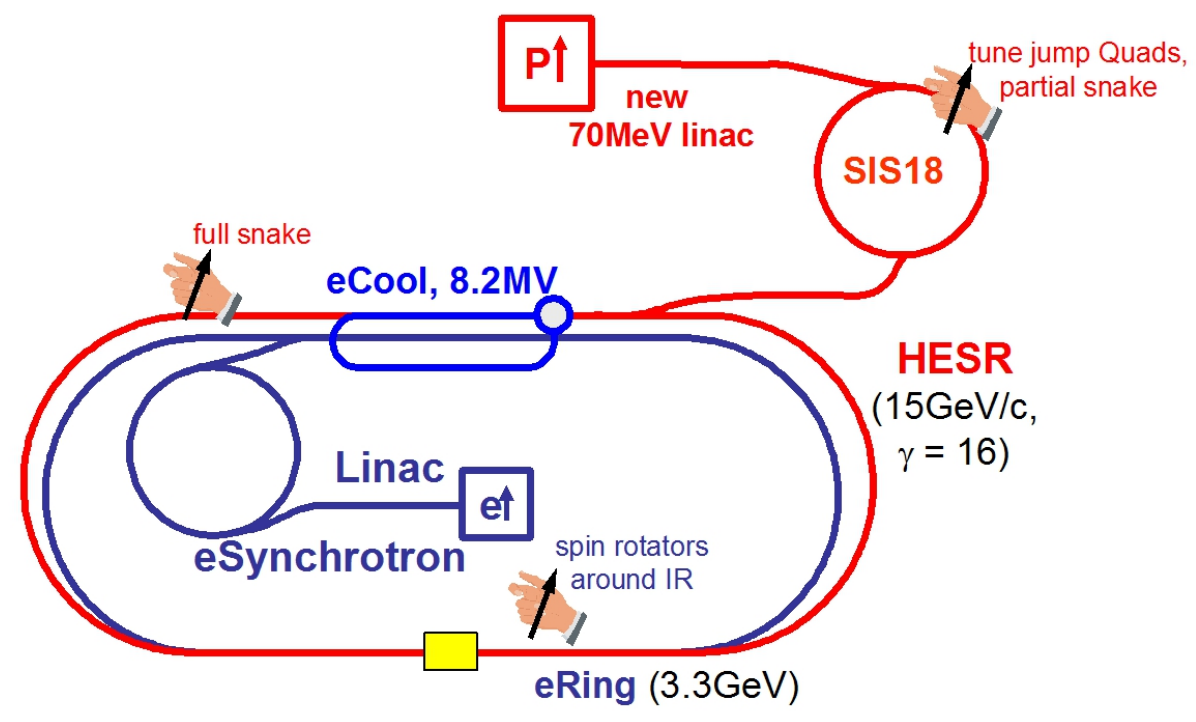

Figure 4: Scheme of ENC at FAIR for electron-proton collisions (not to scale).

\section{Cooler Synchrotron COSY}

At the cooler synchrotron COSY unpolarized and polarized protons and deuterons are accelerated and stored up to a momentum of $3.65 \mathrm{GeV} / \mathrm{c}$. To prepare high precision beams for internal and external experiments two different techniques for beam cooling are utilized: Electron cooling to increase phase space density at injection energy by means of stacking injection together with transverse feedback and stochastic cooling to counteract beam heating of stored ions due to interaction with internal targets. Major accelerator upgrades within the next two years will significantly increase the performance of COSY. Highest precision beams and a significant increase in luminosity can be reached with the implementation of the new $2 \mathrm{MV}$ electron cooler. Utilizing a Siberian snake, longitudinal polarized beams can be provided to internal and external experiments at COSY.

\subsection{MV Electron cooler}

A new $2 \mathrm{MV}$ electron cooler is being built in close collaboration with the Budker Institute for Nuclear Physics and will be installed at COSY in 2011/12 [19]. COSY's luminosity for internal experiments will be increased by more than one order of magnitude. Technical developments for this electron cooler are important steps towards the 4.5(8) MV electron cooler proposed for HESR. The HESR electron cooler layout will also strongly benefit from the experiences of the electron cooler operation at COSY. The measurement of beam cooling forces and other features of magnetized electron cooling at high energies are essential for the planed HESR electron cooler. 
For the startup phase of the HESR this $2 \mathrm{MV}$ electron cooler is well suited for beam cooling and accumulation at injection energy.

\subsection{Siberian snake}

The Siberian snake concept to preserve beam polarization during acceleration has experimentally been validated at the IUCF cooler ring in Bloomington (IN) and successfully implemented in the Relativistic Heavy-Ion Collider RHIC at Brookhaven National Laboratory in Upton (NY). A full Siberian snake rotates the spin of beam particles by $180^{\circ}$ per beam revolution along a horizontal axis (snake axis). If only one full Siberian snake is implemented inside a circular accelerator, the invariant spin axis is located in the horizontal plane around the ring. This is an essential feature to perform spin experiments with longitudinally polarized beams in medium energy circular accelerators. Due to large orbit excursions of the beam in dipole snake magnets at medium energies, a solenoid snake has to be utilized for the COSY energy range. A $5 \mathrm{Tm}$ superconducting solenoid will be installed in the Cooler Synchrotron COSY in 2012/13. Several applications utilizing such a snake system have been proposed:

- Spin-filtering: For optimum polarization buildup in proton (antiproton)-proton collisions along the longitudinal axis at COSY and CERN/AD, integrated field strength of $3.8 \mathrm{Tm}$ is needed for kinetic beam energy of $450 \mathrm{MeV}$.

- Spin physics: For measurements of spin correlation functions with the ANKE detector at COSY, integrated field strength of $3.3 \mathrm{Tm}$ is needed for a kinetic beam energy of $353 \mathrm{MeV}$.

- Accelerator physics: A full Siberian snake with an integrated field of $4.7 \mathrm{Tm}$ is able to preserve the polarization up to kinetic beam energies of $625 \mathrm{MeV}$. The depolarization mechanisms caused by snake resonances (higher-order spin resonances) is planned to be studied at COSY in detail. This development is essential to upgrade the planned High-Energy Storage Ring HESR of FAIR at GSI Darmstadt for polarized beams.

- EDM search: A full Siberian snake could also play a central role in order to perform an EDM precursor experiment at COSY [20] and prepare the spin direction for a dedicated EDM storage ring before injection.

\section{Electric Dipole Moment Storage Ring}

A novel method has been propose within an international collaboration using dedicated storage rings to determine the electric dipole moment of protons and deuterons with a one sigma sensitivity of $10^{-29} \mathrm{e} \cdot \mathrm{cm}$. per $10^{7} \mathrm{~s}$ running time [21]. At this level it will be the best experiment among current and currently planned EDM experiments. The main bending field for the proposed proton EDM experiment has to be purely electrostatic whereas combined electrostatic and magnetic field deflectors are essential to perform a deuteron or helium-3 EDM experiment (see Fig. 5), which is complimentary to the proton EDM measurement. The maximum required electric field gradient for all storage ring options is not larger $17 \mathrm{MV} / \mathrm{m}$ with a magnetic field up to $0.5 \mathrm{~T}$. In the FNAL Tevatron the electrostatic separators operate reliably at roughly the same voltage level for $5 \mathrm{~cm}$ 


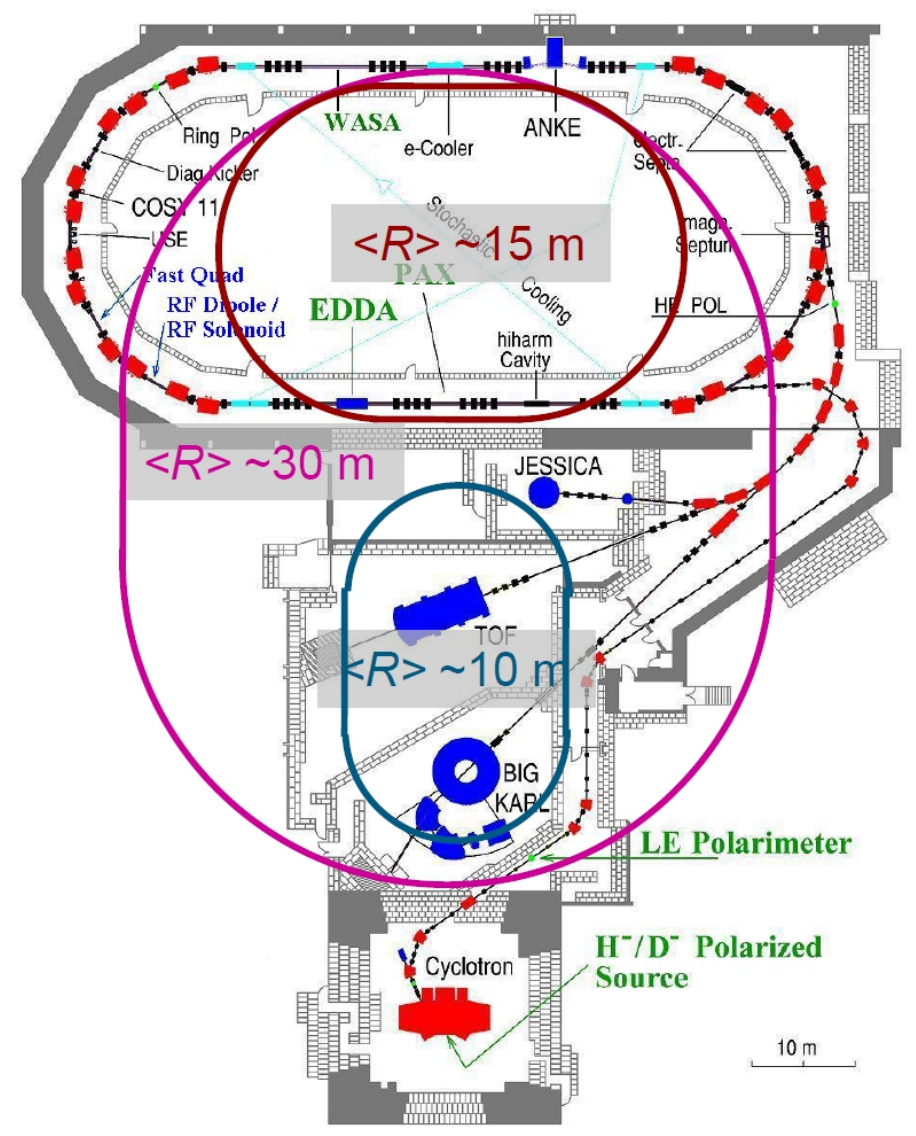

Figure 5: EDM storage ring options at COSY.

plate separation. The proposed techniques are tested and perfected by performing decisive beamand spin dynamics studies as well as crucial hardware tests at RHIC, KVI (Kernfysisch Versneller Instituut, Groningen) and COSY, where polarized hadron beams are available.

\section{Summary/Outlook}

Several hadron storage rings are either successfully operated, under construction or proposed. The FAIR accelerator facility will be realized in six modules, including several hadron storage rings. In the FAIR start version the Collector Ring CR and the High-Energy Storage Ring HESR will be built. The design work of the HESR is in the final stage and the construction phase can start together with FAIR construction. The two polarized colliders proposals - PAX and ENC - are natural expansions of FAIR for spin physics. They could substantially extent the FAIR physics program within the next decade. The Cooler Synchrotron COSY has reached his ultimate performance and a major accelerator upgrade will further strengthen the COSY physics program. COSY is also the ideal machine for beam dynamics studies with respect to HESR and EDM precursor experiments. COSY will finally also be a well-suited injector for a dedicated light-ion EDM ring at FZ Jülich. 


\section{References}

[1] An International Accelerator Facility for Beams of Ions and Antiprotons, FAIR Baseline Technical Report, GSI Darmstadt, ISBN 3-9811298-0-6 (2006).

[2] FAIR - Facility for Antiproton and Ion Research, Green Paper - The Modularized Start Version, GreenPaper, October 2009, see http://www.fair-center.de/.

[3] PANDA Collaboration, Physics Performance Report for PANDA, Strong Interaction Studies with Antiprotons, High Energy Physics - Experiment [hep-ex], arXiv:0903.3905v1.

[4] A. Lehrach et al., Beam Dynamics of the High-Energy Storage Ring (HESR) for FAIR, International Journal of Modern Phys. E (IJMPE) Vol. 18 Issue: 2, 420 (2009).

[5] D.M. Welsch et al., Investigation and Optimization of Transverse Non-linear Beam Dynamics in the High-Energy Storage, Proc. of IPAC10, Kyoto, Japan, THPE063, 4659 (2010).

[6] F. Hinterberger, Ion Trapping in the High-Energy Storage Ring HESR, Bericht des Forschungszentrums Jülich, Jül-4343 (2011).

[7] H. Stockhorst et al., Stochastic Momentum Cooling Experiments with a Barrier Bucket Cavity and Internal Targets at FAIR, Proc. of IPAC10, Kyoto, Japan, MOPD068, 846 (2010).

[8] T. Katayama et al., Beam Accumulation with Barrier Voltage and Stochastic Cooling, Proc. of IPAC10, Kyoto, Japan, MOPD065, 837 (2010).

[9] H. Soltner et al., Magnetic-Field Calculations for the Magnets of the High-Energy Storage Ring (HESR) at FAIR, Proc. of PAC09, Vancouver, BC, Canada, MO6PFP016, 166 (2009).

[10] U. Bechstedt (FZ Jülich), private communication.

[11] R. Stassen et al., COSY as Ideal Test Facility for HESR RF and Stochastic Cooling Hardware, Proc. of PAC09, Vancouver, BC, Canada, TU5PFP022, 861 (2009).

[12] F. Rathmann et al., Phys. Rev. Lett. 93, 224801 (2004).

[13] Antiproton-Proton Scattering Experiments with Polarization, Technical Proposal, PAX Collaboration, January 2006, http://www2.fz-juelich.de/ikp/pax/portal/documents/proposals/files/techproposal20060125.pdf.

[14] F. Bradamante et al., Conceptual Design for a Polarized Proton-Antiproton Collider Facility at GSI, INFN-TC-05-14 (2005), see arXiv.org: physics/0511252.

[15] A. Jankowiak et al., Concept of a polarized electron-nucleon collider utilizing the HESR storage ring at GSI/FAIR, Proc. of PAC2009 Vancouver (BC) Canada, http://trshare.triumf.ca/pac09proc/proceedings/papers/we6pfp063.pdf.

[16] O. Boldt et al., Investigation of various electron ring concepts for the ENC with regard to depolarising effects, J. Phys.: Conf. Ser. 295012157 (2011)

[17] A. Lehrach et al., The ENC@FAIR Project, J. Phys.: Conf. Ser. 295012156 (2011).

[18] A. Lehrach et al., Polarized Beams in the High-Energy Storage Ring of the future GSI Project, Proc. of the 16th International Spin Physics Symposium SPIN 2004, Trieste, World Scientific, 742 (2005), ISBN 9812563156.

[19] J. Dietrich et al., Status of the $2 \mathrm{MeV}$ Electron Cooler for COSY/ HESR, Proc. of IPAC10, Kyoto, Japan, MOPD067, 843 (2010).

[20] F. Rathmann, N.N. Nikolaev, this proceedings.

[21] For further reading see http://www.bnl.gov/edm/ 